\title{
EVALUATION OF DEEP ANTERIOR LAMELLAR KERATOPLASTY VERSUS INTRACORNEAL RING SEGMENTS IN TREATING MODERATE KERATOCONUS
}

\author{
Mohammed Alsaied Alnaimy", Abdelmonem Alsyed Abou-sharkh, Mahmoud Nasr Al-deeb and \\ Ayman Mohammed Abdelrahman \\ Ophthalmology Department, Faculty of Medicine, Zagazig University, Zagazig, Egypt.
}

\section{*Corresponding author:}

Mohammed Alsaied Alnaimy, Mobile: 01226043131

Email:moh_alnaimy@hotmail.com
Background: Keratoconus can cause gradual distortion of vision due to progressive myopia and irregular astigmatism. keratoconus is classified into four stages. The treatment is stage specific starting from glasses or hard contact lenses in the very early stages passing to intra corneal ring segments(ICRS) with or without collagen cross linking(CXL) in the second stage then deep anterior lamellar keratoplasty (DALK) or intra corneal ring segments in the third stage ending with penetrating keratoplasty in the fourth stage.

Patients and Methods: This study included 50 Patients from attendants to outpatient clinic Ophthalmology Department, Zagazig University. Those patients were diagnosed as moderate keratoconus according to modified Amsler classification. The patients were divided randomly into 2 equal groups each one involved 25 eyes. The patients of the first group had DALK using the double bubble modification of the Big Bubble (BB) technique while patients of second group had femto assisted intrastromal corneal ring segment (KERA) implantation. The patients were followed up for 12 months. This prospective randomized interventional study aimed to evaluate and compare intracorneal ring segments (ICRS) versus deep anterior lamellar keratoplasty (DALK) in treating moderate keratoconus. A complete ophthalmic examination was performed, including visual acuity, refraction, and keratometric readings.

Results: The primary outcome measure in this study was the best corrected visual acuity (BCVA) at the end of follow up period which is 12 months while the secondary outcome measures were the uncorrected visual acuity (UCVA), mean spherical and mean $\mathrm{K}$ value. In the first group (DALK group) the mean UCVA preoperatively was $0.05 \pm 0.001$ and mean $\mathrm{BCVA}$ preoperatively was $0.08 \pm$ 0.001 while postoperatively the mean UCVA was $0.1 \pm 0.02$ and mean BCVA postoperatively in the $3^{\text {rd }}$ month was $0.25 \pm 0.02$ and in the $6^{\text {th }}$ month visit was $0.5 \pm 0.02$. In the second group (ICRS group), the mean preoperative uncorrected visual acuity (Pre UCVA) was $0.06 \pm 0.001$ while mean preoperative bestcorrected visual acuity (Pre BCVA) was $0.08 \pm 0.001$. The mean postoperative uncorrected visual acuity (post UCVA) was $0.21 \pm 0.02$ while the mean postoperative best-corrected visual acuity (post BCVA) was $0.4 \pm 0.01$. All patients of both group had progressive visual improvement in their BCVA compared to the preoperative one. In both groups also, the spherical equivalent, spectacle astigmatism and mean central $\mathrm{K}$ value showed significant improvement postoperative compared to preoperative.

Conclusion: Both femtoassisted intracorneal ring segments and deep anterior lamellar keratoplasty are safe and effective surgical alternatives in treating patients with moderate keratoconus. Both of them lead to progressive improvement in the visual outcome of keratoconus patients. Achieving the deepest possible interface to reduce scarring, achieve a posterior layer of uniform thickness, perform smooth surface sectioning of both the graft and bed, make the graft tissue of appropriate thickness, obtain the highest quality donor material, insure good coaptation of the edges and uniform traction of the sutures, and make sure there is perfect cleanliness of the interface are the factors of obtaining best visual results after DALK procedure.

Key notes: moderate keratoconus, deep anterior lamellar keratoplasty, intracorneal ring segment.

\section{INTRODUCTION}

K eratoconus is a degenerative disorder of the eye in which structural changes within the cornea cause it to thin and change to a more conical shape than its normal gradual curve. causing gradual distortion of 
vision due to progressive myopia and irregular astigmatism and typically diagnosed in the patient's adolescent years ${ }^{[1]}$. Because surgical treatment of keratoconus is stage specific ${ }^{[2]}$, there are many classifications that have been developed for tracking disease severity in keratoconus. keratoconus according to modified Amsler Krumeich has four grades. Keratoconus suspect there is eccentric steepening, myopia, induced astigmatism, or both $-5.00 \mathrm{D}$, mean central $\mathrm{K}$ readings $48.00 \mathrm{D}$, mild keratoconus there is myopia, induced astigmatism, or both from 5.00 to $-6.00 \mathrm{D}$, mean central $\mathrm{K}$ reading 51.00 $\mathrm{D}$, absence of scarring, minimum corneal thickness 400 micron, moderate keratoconus :Myopia, induced astigmatism, or both from6.00 to $-10.00 \mathrm{D}$, mean central $\mathrm{K}$ readings $53.00 \mathrm{D}$, absence of scarring, minimum corneal thickness 300 to 400micron and in severe keratoconus, Refraction not measurable, mean central K readings $55.00 \mathrm{D}$, Central corneal scarring, Minimum corneal thickness 200 micron ${ }^{[3]}$.

In very early keratoconus, spectacles or soft contact lenses are sufficient to correct the mild astigmatism. As the condition progresses, these may no longer provide the patient with a satisfactory degree of visual acuity, and most clinical practitioners will move to manage the condition with rigid contact lenses, known as rigid, gaspermeable, (RGP) lenses. RGP lenses provide a good level of visual correction, but do not arrest progression of the condition [4]. Implantation of intracorneal ring segments in moderate to severe $\mathrm{KC}$ is safe and effective [5]. It is recent surgical alternative to corneal transplantation. A small incision is made in the periphery of the cornea either mechanically or by the assist of femtolaser and one or two thin arcs of polymethyl methacrylate are slid between the layers of the stroma on either side of the pupil.The segments push out against the curvature of the cornea, flattening the peak of the cone and returning it to a more natural shape ${ }^{[6]}$. Between $11 \%$ and $27 \%$ of cases of keratoconus will progress to a point where vision correction is no longer possible, thinning of the cornea becomes excessive, or scarring as a result of contact lens wear causes problems of its own and transplantation becomes a must ${ }^{\text {[7]. }}$

Deep anterior lamellar keratoplasty (DALK) procedure for the treatment of keratoconus in patients with moderate to severe disease and steep curvature seems to provide similar efficacy to penetrating keratoplasty and may decrease the risk of immune rejection ${ }^{[8]}$. In a DALK graft, only the outermost epithelium and about $80 \%$ of corneal stroma are replaced; the patient's rearmost endothelium layer and the Descemet's membrane are left, giving some additional structural integrity to the transplanted cornea ${ }^{[9]}$. Comparisons indicate that outcomes are similar to penetrating keratoplasty in terms of visual acuity and astigmatism, with about $80 \%$ of patients achieving a best-corrected visual acuity of $20 / 40$ or greater in most series [10] Technology assessment by the American Academy of Ophthalmology concluded that DALK is equivalent to penetrating keratoplasty in terms of refractive error, and is superior for preservation of endothelial cell density. They believe that wherever feasible, DALK should be performed as the standard of care surgery when keratoplasty is required in keratoconus ${ }^{[11]}$.

\section{PATIENTS AND METHODS}

Before initiating this study, the protocol, the informed consent form and any other written information to be given to patients was reviewed and approved by the Ethics Committee of the Zagazig University Hospital. 50 eyes of 43 Patients with moderate keratoconus were enrolled in this study from attendants to outpatient clinic, ophthalmology department, Zagazig University. The inclusion criteria were Patients with moderate keratoconus (according to modified Amsler Krumeich classification) who fulfilled the following criteria: myopia, induced astigmatism, or both from -6.00 to -10.00 spherical equivalent, mean central $\mathrm{K}$ readings more than $53.00 \mathrm{D}$ and less than $60.00 \mathrm{D}$, Minimum corneal thickness 400 micron, absence of corneal scaring. The exclusion Criteria were Previous corneal surgery, concurrent posterior segment 
disease, patients with myopia, induced astigmatism, or both less than $-6.00 \mathrm{D}$ or more than $10 \mathrm{D}$, corneal thickness less than 400 micron and/ or corneal scaring, patients with corneal dystrophy, autoimmune or collagen disease, vernal keratoconjunctivitis to avoid recurrence, glaucoma or cataract, patients with history of acute hydrops, those in whom complications occurred like large descement perforation and we mentioned that. All patients were subjected to the following: (Preoperative assessment) history taking, complete general medical assessment searching for systemic associations of keratoconus like Marfan syndrome, Down syndrome, mitral valve prolapse, osteogenesis imperfect or atopy and complete general ophthalmic examination was done in all patients including: Slit-lamp evaluation: For signs of Keratoconus like stromal thinning, conical protrusion, Fleischer's ring, Vogt's striae, and exclude Corneal scarring. Retinal evaluation: by indirect ophthalmoscope to exclude retinal pathology and direct ophthalmoscope to detect oil droplet sign. Uncorrected visual acuity (UCVA), Refraction by retinoscopy and subjective refraction (both Cycloplegic and Manifest refraction), Best corrected visual acuity (BCVA) using Snellen chart and Pentacam for detecting the average $\mathrm{K}$ reading, thinnest corneal location, average corneal thickness at 6 and $8 \mathrm{~mm}$ corneal diameter (to determine the depth of trephination and number of trephine quarter rotations intra operative). Primary outcome measure was the best corrected visual acuity (BCVA) at the end of follow up period which is 12 months and secondary outcome measures were uncorrected visual acuity (UCVA), mean spherical equivalent and Pentacam 3-6 months after surgery (for suture manibulation in the first group and monitoring the $\mathrm{K}$ value in the both groups to compare it with the preoperative one).

The patients were divided randomly into 2 equal groups each one involved 25 eyes. In the first group (DALK group), the patients of this group had DALK using the double bubble modification of the Big Bubble (BB) technique as described by
Foroutan and Dastjerdi (2007). All cases were operated by a single experienced anterior segment surgeon. The recipient operative procedure was done first before stripping the descement membrane from the donor so as not to lose the graft if large DM perforation occurred and the surgeon decided to covert to PKP and it was done as follows: All patients were operated under general anesthesia under complete aseptic conditions. The technique started with marking the center of the cornea then 8 or 16 radial marker was used to help postoperative suturing. Trephination of the cornea was done trying to reach about $80 \%$ of the corneal thickness depending on preoperative pentacam. Peripheral paracentesis is done away from the edge of trephination with curved micro vitroretinal blade (MVR) and small bubble of air was injected into the anterior chamber. Twenty seven gauge cannula was introduced into the paracentral corneal stroma for about $4 \mathrm{~mm}$ from the edge of trephination and a second air bubble was injected into the corneal stroma trying to achieve single large sufficient big bubble. After adjusting the intraocular pressure, superficial lamellar keratectomy was done. Releasing of air was done with the sharp tip of MVR after putting a drop of healon to slow the release of air, and then the residual corneal stroma was divided into 4 quadrants with the help of right and left transplantation scissors and then removed baring the descemet's membrane. The donor graft was prepared by removing the descemet membrane with micosponge after flooding it with trypan blue dye. Trephination of the donor graft was done 0.25 to $0.50 \mathrm{~mm}$ larger than the recipient bed to avoid postoperative astigmatism. Suturing of the donor graft to the recipient bed was done in all cases using 16 interrupted sutures. All eye patches were replaced in the first day and patching continued for one week also the contact lens removed four days postoperative. All eyes were treated with topical moxifloxacin ophthalmic solution every 2 hours in the first two days, five times a day for three weeks, then 3 drops per day for another month. 
Topical steroid in the form of prednisolone acetate eye drops $1 \%$ was applied every 2 hours in the first 3 days and 5 to 6 times a day for the next month then 4 times for the subsequent two months and the patient was maintained on 3 times daily for another 3 months. Systemic steroids and systemic antibiotics were given routinely in all patients for two weeks. One drop per day cycloplegic for aweek was used. All eyes are examined at the first day postoperative by slit -lamp examination to test graft clarity, corneal sutures, wound coaptation or leakage, interface haze, and double $\mathrm{AC}$, state of corneal epithelium and signs of infection, inflammation or other surgical complications. All eyes are examined in the first and third day post-operative then every week for the first 2 months then every month during the postoperative period for refraction and Best spectacle corrected visual acuity (BSCVA) using Snellen's eye chart was measured. Pentacam imaging was done to check for corneal irregularities, astigmatism and selective sutures removal and $K$ value recording. Astigmatism more than 4 Diopter was treated in the third month follow up visit by selective suture removal. Otherwise no obligatory suture removal was planned for all cases except if there was complication from suture and needed to be removed.

In the second group (ICRS group); the 25 eyes of 18 patients of this group had femto assisted intrastromal corneal ring segment (Kera) implantation. Either one or 2 segments was chosen according to the Nomogram of Kera ring. The patients were followed up for 12 months. All procedures were performed under topical anesthesia. Skin sterilization was done by Betadine $10 \%$ solution. Wire eye speculum placed for widening of the palpebral fissure. Ocular irrigation was done with Betadine 5\% solution. A disposable suction ring was placed and centered with respect to the pupil center at the area of implantation. The tunnel was done at $80 \%$ of the corneal thickness with the aid of femtosecond laser. This beam formed cavitations, microbubbles, of carbon dioxide and water vapor by photodisruption, and the interconnecting series of these bubbles formed a dissection plane. An inner diameter of $4.8 \mathrm{~mm}$ and outer diameter of $5.4 \mathrm{~mm}$ was programmed with the laser software giving a tunnel width of $0.6 \mathrm{~mm}$, which is equivalent precisely to the segment width and with an incision length of $1.4 \mathrm{~mm}$ was performed on the steepest axis of pentacam. In all eyes, the power used to create the tunnel and the incision was $5 \mathrm{~mJ}$. The procedure lasted approximately 10 seconds. Immediately after clearance of the gas bubbles, the intracorneal ring segments was implanted easily under full aseptic conditions with a special forceps and the segments were placed in the final position with a Sinskey hook through a dialing hole at both ends of the segment. No sutures were placed. A soft bandage contact lens was applied for 48 hours. Postoperative treatment included combination of antibiotic and steroid eye drops 4times daily for 1 week. Patients were instructed to avoid eye rubbing. Contact lens was removed after 48 hours. Patients were followed up at one day, third day, one week, two weeks, one month, three months, and six months postoperatively. Each visit, the patients were asked about notice of vision improvement, any complaints of photophobia glare and halos, ophthalmological examination with emphasis on visual acuity(Uncorrected and best corrected visual acuity), refraction(manifest refraction and cycloplegic refraction by auto refractometer. Slit lamp examination for clarity of corneal center checking the incision, Segments position, asymmetry and depth, any signs of complications (superficial placement, decentration, migration, extrusion, and/or infection). Corneal Imaging: Pentacam with Scheimpflug imaging done 3 to 6 month post operatively to monitor the regularity of the cornea and the average $\mathrm{K}$ value.

Preoperative data

\section{RESULTS}

\section{Demographic data:}

First group (DALK group): In 1 eye (4\%), the procedure was converted to penetrating keratoplasty $(\mathrm{PK})$ intraoperatively because of DM macroperforations. One patient (one eye) (4\%) was lost from the follow up 2 weeks post-operative due to travelling abroad. Another patient (one eye) (4\%) was 
exposed to severe post traumatic graft infection 3 weeks post-operative. Therefore, data for 22 eyes of 22 patients were included for analysis. Thirteen patients were female and nine were males $(50 \%)$. The age of the patients ranged from 14 to 35 years with the mean age was $25.6 \pm 1.9$ years (Mean \pm standard error "SE" was used in the results).

Second group (ICRS group): Eleven females $(61.2 \%)$ and seven males (38.8\%). seven patients were bilateral (3 males and 4 females). Six patients had KC in their RT eye while 5 patients had in their LT eye. The mean age of patients was 25.5 years with a range from 18 to 34 years.

\section{Visual acuity (Table 1):}

First group (DALK group): mean preoperative uncorrected visual acuity (Pre UCVA) was $0.05 \pm 0.001$ while mean preoperative best-corrected visual acuity (Pre BCVA) was $0.08 \pm 0.001$. Second group (ICRS group): mean preoperative uncorrected visual acuity (Pre UCVA) was $0.06 \pm 0.001$ while mean preoperative best-corrected visual acuity (Pre BCVA) was $0.08 \pm 0.001$.

Spherical equivalent, spectacle astigmatism and topographic cylinder (Table 1):

First group (DALK group): The mean preoperative spherical equivalent was -6.58 D. \pm 0.26 with a range between -4.75 to -8.00
D. The mean preoperative spectacle astigmatism was $-4.48 \pm 0.28$ with a range between -2.50 to $-6.25 \mathrm{D}$. The mean preoperative topographic cylinder was $-4.02 \pm$ 0.37 with a range between -1.50 to $-6.60 \mathrm{D}$. Second group (ICRS group): The mean preoperative spherical equivalent was -6.24 D. \pm 0.26 with a range between -4.50 to -8.50 D. The mean preoperative spectacle astigmatism was $4.43 \pm 0.27$ with a range between -2.43 to $-6.35 \mathrm{D}$. The mean preoperative topographic cylinder was $3.96 \pm$ 0.36 with a range between -1.75 to $-6.50 \mathrm{D}$

\section{$K$ values (Table 1):}

First group (DALK group): The mean preoperative central corneal curvature (average $\mathrm{K}$ ) was $54.89 \mathrm{D} \pm 0.64$ with a range between $49.00 \mathrm{D}$ to 58.00 . The mean preoperative $\mathrm{K} 1$ was $50.81 \mathrm{D} \pm 0.57$ with a range between $48.90 \mathrm{D}$ to $56.10 \mathrm{D}$. The mean preoperative $\mathrm{K} 2$ was $56.39 \mathrm{D} \pm 0.71$ with a range between 50.20D to 60.00D. Second group (ICRS group): The mean preoperative central corneal curvature (average K) (Pre Kaverage) was $53.24 \mathrm{D} \pm 0.57$ with a range between $49.00 \mathrm{D}$ to 57.80 . The mean preoperative $\mathrm{K} 1$ was $51.38 \mathrm{D} \pm 0.57$ with a range between $48.30 \mathrm{D}$ to $56.70 \mathrm{D}$. The mean preoperative $\mathrm{K} 2$ was $55.28 \mathrm{D} \pm 0.66$ with a range between $50.20 \mathrm{D}$ to $59.80 \mathrm{D}$.

Table 1: Preoperative Data of both groups.

\begin{tabular}{cccc}
\hline \multirow{2}{*}{ Parameters } & \multicolumn{2}{c}{ Preoperative data of both groups } & \multirow{2}{*}{ Significance } \\
\cline { 2 - 3 } & DALK data & ICRS data & $\mathrm{P}>0.05$ \\
\hline UCVA & 0.05 & 0.06 & $\mathrm{P}>0.05$ \\
\hline BCVA & 0.08 & 0.08 & $\mathrm{P}>0.05$ \\
\hline K 1 & 50.81 & 51.38 & $\mathrm{P}>0.05$ \\
\hline K2 & 56.39 & 55.28 & $\mathrm{P}>0.05$ \\
\hline Average K & 54.89 & 53.24 & $\mathrm{P}>0.05$ \\
\hline S EQ & -6.58 & -6.24 & $\mathrm{P}>0.05$ \\
\hline TOPO. CYL & -4.02 & -3.96 & $\mathrm{P}>0.05$ \\
\hline SPE. CYL(D) & -4.48 & -4.43 & \\
\hline
\end{tabular}

$\overline{\text { From Table (1), data showed that there was no statistically significant difference between the }}$ preoperative data in both groups.

\section{Intra operative data:}

First group (DALK group): Twelve $(54.55 \%)$ had single successful air bubble from the first injection. Seven $(31.81 \%)$ eyes required repeated air injection twice or three times to get a single perfect air bubble. Three $(13.64 \%)$ eyes failed to get a single air bubble even after repeated air injection in the stroma in different location with opacification of the whole stroma and 
shifting to layer-by-layer manual stromal dissection technique was done removing the stroma layer by layer till reaching the descement membrane (DM).

One $(4.5 \%)$ eye had an accidental microperforation and was noted by the escape of aqueous from the anterior chamber. It was differentiated from macroperforation by injecting air inside the $\mathrm{AC}$ and was maintained inside the anterior chamber (AC). In this patient, Healon was used to push the DM away down and the dissection was completed across the cornea. Later after securing the graft in the recipient bed, injection of air was done through the limbal paracentesis to oppose the Descemet's membrane against the deep stroma aiming to avoid the double anterior chamber. One eye (1/25) (4\%) required conversion to a PK intraoperatively because of DM macroperforation due to accidental opening of the The anterior chamber during trephination .so we shifted to PK with aid of the curved corneal scissors which were introduced through the defect after injecting Healon to reform the anterior chamber. This eye was excluded from the analysis (Table. 2).

Table 2: Introperative data of the first group.

\begin{tabular}{lcc}
\hline \multicolumn{1}{c}{ Introperative data } & Number & $(\%)$ \\
\hline Successful single bubble from first injection & $12 / 22$ & $(54.55 \%)$ \\
\hline - Multiple air injection before successful single bubble. & $7 / 22$ & $(31.81 \%)$ \\
\hline $\begin{array}{l}\text { Failed air bubble and shifting to layer-by-layer manual stromal } \\
\text { dissection }\end{array}$ & $3 / 22$ & $(13.64 \%)$ \\
\hline DM micro perforation & $1 / 22$ & $(4.5 \%)$ \\
\hline Conversion to PK & $1 / 25$ & $(4 \%)$ \\
\hline
\end{tabular}

Second group (ICRS group): The intracorneal ring segment(s) was implanted easily under full aseptic conditions with a special forceps and the segments were placed in the final position with a Sinskey hook through a dialing hole at both ends of the segment and no sutures were placed. No intraoperative complications like segment loss, anterior chamber perforation, difficult insersion or false tracking.

\section{Postoperative Data (Table 3): \\ Visual acuity:}

First group (DALK group): All patients had progressive visual improvement in their BCVA compared to the preoperative one at the end of the follow up period. Mean UCVA preoperatively was $0.05 \pm 0.001$ while mean UCVA postoperatively $0.1 \pm 0.02$. Mean BCVA preoperatively was $0.08 \pm 0.001$ while mean BSCVA postoperatively in the $3^{\text {rd }}$ month was $0.25 \pm 0.02$ and in the $6^{\text {th }}$ month visit was $0.5 \pm 0.02$. The uncorrected visual acuity increased postoperatively by a mean of $100 \%$ and the best-corrected visual acuity by a mean of $525 \%$. From the above mentioned UCVA and BCVA changes, highly significant improvement in visual acuity was noted $(\mathrm{P}<0.001)$. Second group (ICRS group): mean preoperative uncorrected visual acuity (Pre UCVA) was $0.06 \pm 0.001$ while mean preoperative best-corrected visual acuity (Pre BCVA) was $0.08 \pm 0.001$. All patients had progressive visual improvement in their BCVA compared to the preoperative one at the end of the follow up period. The mean postoperative uncorrected visual acuity (Post UCVA) was $0.21 \pm 0.02$ with. The mean postoperative best-corrected visual acuity (Post BCVA) was $0.43 \pm 0.01$. From the above mentioned BCVA changes, highly significant improvement in visual acuity is noted $(\mathrm{P}<0.001)$. The uncorrected visual acuity increased postoperatively by a mean of $233 \%$ and the best-corrected visual acuity by a mean of $400 \%$. The UCVA improved by 1-2 lines in 5 eyes $(20 \%)$ and more than 2 lines in 20 eyes $(80 \%)$ for a total improvement in the whole 25 eyes included in the study. None showed the same preoperative UCVA $(0 \%)$ and none worsened (0\%). The BCVA improved by 1-2 lines in 7 eyes (28\%) and more than 2 lines in 18 eyes $(72 \%)$ for a total improvement in the whole 25 eyes included in the study. None showed the same 
preoperative BCVA $(0 \%)$ and none worsened $(0 \%)$.

Efficacy index $=$ post op. UCVA $(0.21) /$ pre op BCVA $(0.08)=(2.5)$

Safety index $=$ post op BCVA (0.4) / pre op BCVA $(0.08)=(5)$.

Table 3: Postoperative Data of both groups.

\begin{tabular}{cccc}
\hline \multirow{2}{*}{ Parameters } & \multicolumn{2}{c}{ Postoperative data of both groups } & \multirow{2}{*}{ Significance } \\
\cline { 2 - 3 } & Dalk data & ICRS data & $\mathrm{P}<0.01$ \\
\hline UCVA & 0.10 & 0.21 & $\mathrm{P}<0.05$ \\
\hline BCVA & 0.50 & 0.43 & $\mathrm{P}>0.05$ \\
\hline K 1 & 43.12 & 46.23 & $\mathrm{P}>0.05$ \\
\hline K2 & 47.25 & 49.52 & $\mathrm{P}>0.05$ \\
\hline Average K & 45.16 & 47.78 & $\mathrm{P}>0.05$ \\
\hline S EQ & -2.64 & -2.30 & $\mathrm{P}>0.05$ \\
\hline TOPO. CYL & 2.89 & 3.09 & $\mathrm{P}<0.05$ \\
\hline SPE. CYL(D) & 2.67 & 2.18 & \\
\hline
\end{tabular}

\section{Spherical equivalent, Topographic astigmatism and Refraction:}

First group (DALK group): The mean preoperative spherical equivalent was -6.58 D. \pm 0.26 which decreased postoperatively to -2.64 D. \pm 0.23 that was statistically significant with a P-value $<0.01$. The spherical equivalent decreased postoperatively by a mean of $59.87 \%$. The mean preoperative spectacle astigmatism was $4.48 \pm 0.28$ with topographic cylinder $4.02 \pm$ 0.37 which decreased postoperatively to 2.67 \pm 0.30 and $2.89 \pm 0.23$ respectively. That was statistically significant with a P-value $<0.01$. The spectacle astigmatism decreased postoperatively by $40.40 \%$ and the topographic cylinder by $28.10 \%$. Second group (ICRS group): The mean preoperative spherical equivalent was -6.24 D. \pm 0.26 which decreased postoperatively to -2.30 D. \pm 0.45 that was statistically significant with a Pvalue $<0.01$. The spherical equivalent decreased postoperatively by a mean of 63.14 $\%$. The mean preoperative spectacle astigmatism was $4.43 \pm 0.27$ with topographic cylinder $3.96 \pm 0.36$ which decreased postoperatively to $2.18 \pm 0.16$ and $3.09 \pm 0.35$ respectively. That was statistically significant with a P-value $<0.01$. The spectacle astigmatism decreased postoperatively by $50.79 \%$ and the topographic cylinder by 21.96 $\%$.

\section{K value:}

First group (DALK group): The mean preoperative central corneal curvature (average K) was $54.89 \mathrm{D} \pm 0.64$ with $\mathrm{K} 1$
$50.81 \mathrm{D} \pm 0.57$ and $\mathrm{K} 256.39 \mathrm{D} \pm 0.71$. That decreased postoperatively to average $\mathrm{K} 45.16$ $\mathrm{D} \pm 0.43$ with $\mathrm{K} 143.12 \mathrm{D} \pm 0.67$ and $\mathrm{K} 2$ $47.25 \mathrm{D} \pm 0.45$. That was statistically significant with a $\mathrm{P}$-value $<0.05$. The average central corneal curvature decreased postoperatively by a mean of $17.72 \%$. Second group (ICRS group): The mean preoperative central corneal curvature (average $\mathrm{K}$ ) was $53.42 \mathrm{D} \pm 0.57$ with $\mathrm{K} 1$ $51.38 \mathrm{D} \pm 0.57$ and $\mathrm{K} 255.28 \mathrm{D} \pm 0.66$. That decreased postoperatively average $\mathrm{K} 47.78 \mathrm{D}$ \pm 0.55 with $\mathrm{K} 146.23 \mathrm{D} \pm 0.58$ and $\mathrm{K} 249.52$ $\mathrm{D} \pm 0.56$. That was statistically significant with a P-value $<0.05$. The central corneal curvature decreased postoperatively by a mean of $10.55 \%$.

From Table (3) the data showed that there was statistically significant difference between the first and the second groups regarding the UCVA which was better in the second and the BCVA which was better in the first group $(\mathrm{P}<0.05)$. There was also statistically significant difference between the first and the second group regarding the spectacle cylinder which was better in the second group $(\mathrm{P}<0.05)$. Otherwise no statistically significant difference was found in the other parameters.

\section{Clarity of the cornea:}

First group (DALK group): All eyes suffered from mild to moderate corneal edema in the immediate postoperative period which resolved with medication and time with clear graft and clear interface. 
One patient (1/22) suffered from late onset corneal haze ( 2 years after surgery) together with interface vascularization during the period of follow up. She received topical and systemic steroids for 3 weeks with restoration of the corneal clarity.

Second group (ICRS group): Clear cornea with ICRS in place occurred in all cases of second group during the follow up period.

\section{Postoperative complications:}

First group (DALK group): Folds of DM occurred in $3(13.64 \%)$ eye with no affection on the clarity of the corneal graft or visual function so no interference was needed. Interface haze occurred in $1 / 22$ $(4.5 \%)$ eye of the 3 eyes operated by the layer by layer manual dissection technique in the peripheral part of the graft host bed. Secondary pupillary block glaucoma occurred in one $(4.5 \%)$ eye in the first day postoperative and was managed with antiglaucoma for another day by giving $200 \mathrm{cc}$ Mannitol 20\% solution over 20 minutes, acetazolamide $250 \mathrm{mg}$ twice per day and combigan eye drops twice. In the second day the intraocular pressure did not decrease significantly, so the patient was reintroduced to the operating room and under complete aseptic condition with topical anesthesia, some of the air in the anterior chamber was removed through the paracentesis. Loose stitch developed in 15 eyes $(68.18 \%)$ eyes and removed whenever detected. If sutures were removed in the first month, it was taken again and this occurred in two cases only $(9.1 \%)$. If detected more than three months, it was removed. Stitch related in filterates occurred in one eye $(4.5 \%)$ at the end of the first year and removed in the operating room followed by moxifloxacin $0.3 \%$ drops every two hours and fucidic acid ointment twice daily for two weeks. Suture vascularization was seen in six eyes $(27.3 \%)$ and treated with topical steroids for 3 weeks which showed complete resolution and disappearance of the vessels.

No postoperative graft rejection occurred in our study. Single case of posttraumatic, not postoperative, infection has occurred in our study and excluded from the study. Double
Anterior chamber was not observed at any case. One eye developed complicated anterior polar cataract filling the pupil one month post-operative. He was operated in the $4^{\text {th }}$ month with irrigation aspiration and IOL implantation in the bag. Second group (ICRS group): no postoperative complications encountered in this study during the follow up period. Two eyes showed evidence of progression after the end of follow up period in the form of decrease in the UCVA and BCVA and increasing astigmatism which needed stabilization with cross linking.

\section{DISCUSSION}

Keratoconus is a degenerative disorder of the eye in which structural changes within the cornea cause it to thin and change to a more conical shape than its normal gradual curve causing gradual distortion of vision due to progressive myopia and irregular astigmatism. It is typically diagnosed in the patient's adolescent years (Feder and Kshettry, 2005) [1]. Because surgical treatment of keratoconus is stage specific (Rabinowitz et al, 2005) ${ }^{[2]}$, Modified of Amsler Krumeich has classified KC into four stages (Klin and Augenheilkd, 2010) [3] Keratoconus suspect, mild, moderate and severe. This study included 50 eyes of 43 Patients from attendants to outpatient clinic, department of ophthalmology, zagazig University. It was designed in the form of interventional randomized comparative study (eye not patient randomization as the disease has 4 stages and the patient not mandatory share the same stage in both eyes). The inclusions Criteria: patients with moderate keratoconus according to modified Amsler Krumeich classification (Klin and Augenheilkd, 2010) ${ }^{[3]}$ who fulfilled the following criteria: myopia, induced astigmatism, or both from -6.00 to -10.00 spherical equivalent, mean central $\mathrm{K}$ readings more than $53.00 \mathrm{D}$ and less than $60.00 \mathrm{D}$, minimum corneal thickness $400 \mu$ and absence of corneal scaring. The exclusion Criteria were Previous corneal surgery, concurrent posterior segment disease, patients with myopia, induced astigmatism, or both less than $-6.00 \mathrm{D}$ or more than $10 \mathrm{D}$, corneal thickness less than 400 micron and/ or corneal 
scaring, patients with corneal dystrophy, autoimmune or collagen disease, vernal keratoconjunctivitis to avoid recurrence, glaucoma or cataract, patients with history of acute hydrops, those in whom complications occurred like large descement perforation and we mentioned that. Implantation of intracorneal ring segments in moderate to severe $\mathrm{KC}$ is safe and effective. It is recent surgical alternative to corneal transplantation (Kotb and Hantera, 2013) ${ }^{[5]}$.The segments push out against the curvature of the cornea, flattening the peak of the cone and returning it to a more natural shape (Ruckhofer, 2002) ${ }^{[6]}$. ICRS has the advantages of preserving the central cornea and of being a reversible procedure with a return to preoperative corneal and refractive parameters after ring removal in most cases.In addition; there is excellent corneal tolerance to PMMA rings (Barbara et al., 2005) ${ }^{[12]}$. Femto assisted ICRS implantation is safe, effective, non time consuming with no needs of long learning curve or special set of instruments in comparison to manual ICRS implantation (Kotb and Hantera, 2013) ${ }^{[5]}$. Kera ring segments were used in this study as they were available during the study has different diameters and arc lengthes, triangular with a prismatic effect that can eliminate the halo phenomena. Also, it's inserted in relation to the center of the cornea not the pupil and it is the standared one used nowadays. Deep anterior lamellar keratoplasty(DALK) procedure for the treatment of keratoconus in patients with moderate to severe disease and steep curvature seems to provide similar efficacy to penetrating keratoplasty and has the advantages of decreasing the risk of immune endothelial rejection, not an open sky procedure with its sequele, early suture removal, more blunt traume resistent and earlier visual habilitation (Shi et al., 2010) ${ }^{[8]}$.

DALK using BB technique appears to be safe and effective procedure in treating patients with moderate $\mathrm{KC}$ and have led to significant improvement in the visual outcome for patients with moderate to advanced KC. It enables to achieve bare DM and smooth regular interface which is the main issue in decreasing the risk of interface haze that may occure in DALK surgery (Fogla and Padmanabhan, 2006) ${ }^{[13]}$.

Double bubble modification is very effective method in determining when to finish air injection into the corneal stroma and so, start dissection of the feathery emphysamatous cornea by noticing the shift in the anterior champer bubble (Foroutan and Dastjerdi, 2007) ${ }^{[29]}$.

The patients in this study were randomly assigned into two groups. Each group included 25 eyes. The first group underwent DALK using the double bubble technique while the second underwent femto assisted kera ring implantation using the nomogram of the Kera ring.

The patients were followed up 1 year and the primary outcome measure in this study was the best corrected visual acuity (BCVA) at the end of follow up period while the secondary outcome measures were the uncorrected visual acuity (UCVA), mean spherical and the change in $\mathrm{K}$ value.

Intra operatively, in the first group, Twelve $(54.55 \%)$ had single successful air bubble from the first injection. Seven $(31.81 \%)$ eyes required repeated air injection twice or three times to get a single perfect air bubble. Three $(13.64 \%)$ eyes failed to get a single air bubble even after repeated air injection in the stroma in different location with opacification of the whole stroma and shifting to layer-by-layer manual stromal dissection technique was done removing the stroma layer by layer till reaching the DM. This is comparable to the results of Feizi et al (2010) ${ }^{[18]}$ who evaluated dalk in patients with $\mathrm{KC}$ and achieved bared DM in $65 \%$ of the cases (84 eyes out of 129), whereas in $35 \%$ (45 eyes out of 129) pre-Descemet dissection was performed. This also matches with Fontana etal., (2007) ${ }^{[19]}$ who evaluated DALK in KC patients and Seventy-eight procedures out of eighty one $(96 \%)$ were completed as DALK. Big-bubble was achieved in 50 cases (64\%); 28 (36\%) required manual intrastromal dissection.

One $(4.5 \%)$ eye had an accidental microperforation and was noted by the escape of aqueous from the anterior chamber The rate of DM perforation in patients with 
keratoconus has been reported to be $4 \%$ reported by Feizi et al (2010) ${ }^{[18]}$ and $7.5 \%$ by Kubaloglu et al. (2011) ${ }^{[21]}$. One eye $(1 / 25)$ (4\%) required conversion to a PK intraoperatively because of DM macroperforation due to accidental opening of the The anterior chamber during trephination which is in line with the rate of conversion to PK (from 0 to 4\%) as published by Fogla and Padmanabhan (2006) [13] $(0 \%)$ and Sarnicola et al. (2010) ${ }^{[22]}(0 \%)$, Kubaloglu et al., (2011) ${ }^{[21]}(3 \%)$ and Han et al. (2009) ${ }^{[23]}(4 \%)$.

Intra operatively, in the second group, The intracorneal ring segment(s) was implanted easily under full aseptic conditions with a special forceps and the segments were placed in the final position with a Sinskey hook through a dialing hole at both ends of the segment and no sutures were placed. No intraoperative complications like segment loss, anterior champer perforation, difficult insersion or false tracking. This matches with Coskunseven et al., (2007) ${ }^{[25]}$ who evaluated the femtoassisted ICRS (Kera ring) in treating 50 patients with KC and with Sansanayudh et al., (2010) [27] who evaluated the femtoassisted ICRS (Kera ring) in treating 10 eyes with $\mathrm{KC}$ (moderate to severe $\mathrm{KC}$ ) and in both studies, no intra operative complications were encountered.

Post operatively, in the first group (DALK group), the mean UCVA preoperatively was $0.05 \pm 0.001$ while mean BCVA preoperatively was $0.08 \pm 0.001$ while post operative, all patients had progressive visual improvement in their BCVA compared to the preoperative one. Mean UCVA postoperatively $0.1 \pm 0.02$ and mean BCVA postoperatively in the $3^{\text {rd }}$ month was $0.25 \pm$ 0.02 and in the $6^{\text {th }}$ month visit was $0.5 \pm 0.02$. From the above mentioned BCVA changes, highly significant improvement in visual acuity is noted $(\mathrm{P}<0.001)$. This is matched with Khalid et al., (2010) ${ }^{[15]}$ who conducted a study to evaluate the visual outcome (BCVA) after DALK in KC on 47 patients and $34 / 47$ patients $(73 \%)$ had VA $6 / 12$ to $6 / 9$ at the end of follow up period and concluded that DALK can be considered a suitable technique in patients with $\mathrm{KC}$ providing good visual results. Javadi et al. (2009) [16] evaluated DALKusing $\mathrm{BB}$ technique in patients with moderate to severe $\mathrm{KC}$ on 104 eyes by measuring the BCVA at the end of follow up period and found that there is marked improvement in the VA post operatively $\quad 0.26 \pm 0.2 \quad(20 / 40) \quad \log M A R$ compared to preoperatively1.23 $\pm 0.4(20 / 400)$ $\log$ MAR and concluded that DALK can be safe and effective technique in patients with KC. Fogla (2013) ${ }^{[17]}$ conducted astudy to evaluate DALK in the management of moderate $\mathrm{KC}$ by measuring the BCVA at the end of follow up period and found that there is marked improvement in the VA postoperatively20/30 compared to 20/400 preoperatively. In our study, DALK patients had slightly superior BCVA than those with ICRS reaching 0.5 and 0.4 respectively.

Our results match with Feizi et al., (2010) ${ }^{[18]}$ who evaluated the visual and refractive outcomes after deep anterior lamellar keratoplasty (DALK) using the bigbubble technique in eyes with moderate keratoconus. Atotal of 129 eyes were included. Mean preoperative corrected visual acuity was $1.27 \log$ MAR, increasing to 0.25 $\log$ MAR at final follow-up examination. Postoperative mean spherical equivalent and refractive error were $3.41 \pm 3.1 \mathrm{D}$ and $3.04 \pm$ $2.3 \mathrm{D}$ respectively and were both significantly lower than preoperative and concluded that DALK appears to be a safe and effective procedure for eyes with moderate to advanced keratoconus.

Fontana et al., (2007) ${ }^{[19]}$ evaluated clinical outcomes after deep anterior lamellar Keratoplasty using the big-bubble technique in patients with keratoconus. Baseline mean uncorrected visual acuity was 20/500 pre operative and 20/60 two years after surgery. Average preoperative BSCVA, was 20/100 and 20/30 at the end of follow-up. They concluded that the DALK technique is a valuable treatment in patients with moderate keratoconus. Outcomes of deep anterior lamellar Keratoplasty for Keratoconus during learning curve and advantages of the Big Bubble Technique was evaluated by Smadja et al., (2012) ${ }^{[20]}$ and found that deep lamellar anterior keratoplasty with the BB technique is 
safe and provides very good visual outcomes. At 12 months, the mean best-corrected logarithm of the minimum angle of resolution visual acuity was $0.88 \pm 0.14$ with $93.3 \%$ seeing best-corrected visual acuity 20/30 and $100 \%$ seeing BCVA 20/40. In our study, folds of DM appeared in $1(4.5 \%)$ eye without affection of the corneal clarity. Ünal et al., (2010) ${ }^{[24]}$ recorded it in $(9.5 \%)$ of their patients and attributed this to flattening of the cone by the donor corneal tissue or stretched DM preoperatively which mismatch the size of the transplanted stroma and added that adequate air tamponade at the end of operation may prevent the event.

No postoperative infection occurred in our study (except one case and was related to severe trauma in the early post operative period and it was excluded from our study). This matches with other authors like (Ünal et al., 2010) ${ }^{[24]}$ and Feizi et al. (2010) ${ }^{[18]}$ who evaluated DALK in treating KC patients and they attributed this to the improved and modern preservation methods of the grafts and sterilization techniques used perioperatively including preoperative administration of topical antibiotic eye drops, intraoperative Betadine solution usage for skin and conjunctival sac, draping and isolation of the lids and lashes and postoperative antibiotic regime. Stromal graft rejection didn't occur in our study. The risk of immune-mediated stromal graft rejection was reported between $0 \%$ by Han et al., (2009) [23] and $14 \%$ by Feizi et al. (2010) ${ }^{[18]}$ after DALK surgery.

In the second group (ICRSgroup), the mean preoperative uncorrected visual acuity (Pre UCVA) was $0.06 \pm 0.001$ while mean preoperative best-corrected visual acuity (Pre BCVA) was $0.08 \pm 0.01$. All patients had progressive visual improvement in their BCVA compared to the preoperative one. In the second group.the mean postoperative uncorrected visual acuity (post UCVA) was $0.21 \pm 0.02$ while the mean postoperative best-corrected visual acuity (post BCVA) was $0.4 \pm 0.01$ at the end of follow up.

From the above mentioned BCVA changes highly significant improvement in visual acuity is noted $(\mathrm{P}<0.001)$. This matches with Coskunseven et al., (2007) ${ }^{[25]}$ who evaluated the femtoassisted ICRS (Kera ring) in treating 50 patients with $\mathrm{KC}$ and found that there was significant improvement in both UCVA and BCVA postoperatively compared to preoperatively and concluded that use of femto ICRS implantation in patients with $\mathrm{KC}$ is minimally invasive procedure for improving UCVA and BCVA in patients with $\mathrm{KC}$.

This also matches with Shabayek and Alio (2007) [26] who evaluated the femtoassisted ICRS(Kera ring) in treating 21 patients with moderate to severe $\mathrm{KC}$ and found that there is significant improvement in both UCVA and BCVA postoperatively $(0.3$ and 0.5 respectivly) compared to preoperatively( 0.2 and 0.4 respectivly). This is also matched with Sansanayudh et al., (2010) ${ }^{[27]}$ who evaluated the femtoassisted ICRS (Kera ring) in treating 10 eyes with KC (moderate to severe $\mathrm{KC}$ ) and found that there is significant improvement in both UCVA postoperatively $(0.6 \quad \log \quad$ MAR \pm 0.2 versus $1.19 \pm 0.57 \log \mathrm{MAR})$ and $\mathrm{BCVA}$ $(0.25 \pm 0.15 \log$ MAR versus $0.51 \pm 0.20 \mathrm{log}$ MAR) and recommended use of ICRS in treating moderate to advanced $\mathrm{KC}$.

The UCVA improved by 1-2 lines in 5 eyes (20\%) and more than 2 lines in 20 eyes (80\%) for a total improvement in the whole 25 eyes included in the study. None showed the same preoperative BCVA $(0 \%)$ and none worsened $(0 \%)$. This partially agrees with Coskunseven et al. (2007) ${ }^{[25]}$ who evaluated the femtoassisted ICRS(Kera ring) in treating 50 patients with $\mathrm{KC}$ and found that $39(70 \%)$ eyes gained from 1 to 4 lines in their BCVA but 9 eyes(18\%) showed the same pre operative VA and only 2 eyes(4\%) showed 2 line loss in their BCVA.

In the second group, the mean preoperative spherical equivalent was -6.24 D. \pm 0.26 which decreased postoperatively at the end of follow up to -2.30 D. \pm 0.45 that was statistically significant with a $\mathrm{P}$-value $<$ 0.01 . The spherical equivalent decreased postoperatively after 6 months by a mean of $63.14 \%$. The mean preoperative spectacle astigmatism was $4.43 \pm 0.27$ with topographic cylinder $3.96 \pm 0.36$ which decreased 
postoperatively at the end of follow up to 2.18 \pm 0.16 and $3.09 \pm 0.35$ respectively. That was statistically significant with a P-value $<0.01$. The mean preoperative central corneal curvature (average K) was $53.24 \mathrm{D} \pm 0.57$ with $\mathrm{K} 151.38 \mathrm{D} \pm 0.57$ and $\mathrm{K} 255.28 \mathrm{D} \pm$ 0.66. That decreased postoperatively to (average K) was $47.78 \mathrm{D} \pm 0.55$. The central corneal curvature decreased postoperatively after 6 months by a mean of $10.55 \%$. This matches with Sansanayudh et al., (2010) ${ }^{\text {[27] }}$ who evaluated the femtoassisted ICRS (Kera ring) in treating 10 eyes with moderate $\mathrm{KC}$ and found that there is significant reduction in spherical equivelant (-8.08 to -5.03$)$, astigmatism (-5.05 to -3.90$)$ and mean $\mathrm{K}$ value (57.94 to50.07D). This also agrees with Coskunseven et al., (2013) ${ }^{[28]}$ who evaluated the femtoassisted ICRS (Kera ring) in treating patients with $\mathrm{KC}$ and found that there is stastically significant reduction in both spherical equivalent and refractive error post operatively(-2.49 \pm 2.68$)$ compared to pre operatively $(-5.62 \pm-4.15)$.

In our study, there was no intra or post operative complications encountered during the follow up of the patients underwent femto assisted kera ring implantation. This matches with Coskunseven et al., (2007 and 2013) ${ }^{[25]}$ [28] who evaluated the femtoassisted ICRS (Kera ring) in treating 50 patients with $\mathrm{KC}$ andreached the same results and Shabayek and Alio (2007) ${ }^{[26]}$ who evaluated the femtoassisted ICRS and no complications also occurred during the follow up period.

Regarding the comparison of both groups: there was statististically significant diffrence between the first and the second groups regarding the UCVA which was better in the second and the BCVA which was better in the first group $(\mathrm{P}<0.05)$. There was also statististically significant diffrence between the first and the second group regarding the spectacle cylinder which was better in the second grour $(\mathrm{P}<0.05)$. Other wise no statistically significant diffrence was found in the other parameters between both groups post operative. In comparing our results to Yusuf Ozerturk etal., (2012) [14] who compared these two techniques in moderate $\mathrm{KC}$, we found that his results match with our study as he found that there was statistically significant improvement in all post operative parameters compared to the preoperative ones in both groups (UCVA,BCVA,SE and mean $\mathrm{K}$ value). in comparing the post operative results between the two groups, he found that there was statistically significant diffrence in all post operative parameters (UCVA,BCVA,SE and mean $\mathrm{K}$ value) with the upper hand to the DALK group and explained that by the fact that in DALK he removed the whole diseased tissue. $\mathrm{He}$ concluded that DALK provided greater improvement in the BCVA in moderate $\mathrm{KC}$ but ICRS implantation is an alternative treatment with also satisfactory outcome and less visual impact and this conclusion may be explained by the fact that it was retrospective study so the cases may underwent DALK or ICRS implantation according to the surgeon preference.

\section{CONCLUSION}

Implantation of intracorneal ring segments in moderate to severe keratoconus is safe and effective and can be considered as a surgical alternative to corneal transplantation. In addition, there is excellent corneal tolerance to PMMA rings. Also, with evolution of femto second laser, this procedure takes minutes to finish with minimal intra or post operative complications and very easy short learning curve.

Although a significant improvement was observed in BCVA in the DALK group at the end of follow up, patients with keratoconus are relatively young and may need keratoplasty at any stage of their lives. So, long term graft survival and function are important to keep and maintain the visual ability and quality of life over long period. Hence, we should keep in mind this when deciding to perform keratoplasty for keratoconus patients. On the other hand, ICRS can be removed, exchanged and have no negative impact on the results of subsequent keratoplasty procedures.

On the other hand, beside that the deep anterior lamellar keratoplasty procedure provided results similar to that puplished for penetrating keratoplasty in treating moderate keratoconus, it may decrease the risk of 
immune rejection. Also, the deep anterior lamellar keratoplasty using the big bubble technique appears to be a safe and effective procedure in treating patients with moderate keratoconus. Unfortunately, it has long learning curve, increased incidence of both intra and post operative complications and also needs long follow up and suture manipulations. In this study, DALK patients had slightly superior BCVA than those with ICRS reaching 0.5 and 0.4 respectively. Other many studies reported better visual acuity $(0.8$ or even more) at the end of their follow up. This may be attributed to our relatively short follow up and small group number.

\section{REFERENCES}

1. Feder R, Kshettry P. Non-inflammatory Ectactic Disorders, Chapter 78. In Krachmer J. Cornea. Mosby, 2005.

2. Rabinowitz YS, Bennett ES and Weissman BA. Corneal topography and Clinical Contact Lens Practice. Philadelphia, PA: 2005; 215-232.

3. Klin, M. and Augenheilkd. Keratoconus Stages after Krumeich; J. Ophtha. 2010; 117 (2) : 226-231.

4. Rubinstein MP, Sud S. "The use of hybrid lenses in management of the irregular cornea". Contact Lens \& Anterior Eye,1999; 22 (3): 87-90.

5. Kotb AM, Hantera. M. Efficacy and safety of Intacs SK in moderate to severe keratoconus. 2013; 20(1):46-50.

6. Ruckhofer J. Clinical and histological studies on the intrastromal corneal ring segments (ICRS(R), Intacs(R). Klinische Monatsblätter für Augenhei lkunde (in German) 2002; 219 (8): 557-74.

7. Barr JT, Wilson BS, Gordon MO. Estimation of the incidence and factors predictive of corneal scarring in the Collaborative Longitudinal Evaluation of Keratoconus (CLEK) Study. Cornea (2006): 25 (1): 16-25

8. Shi W, Li S, Gao H, Wang T, Xie L. Modified deep lamellar keratoplasty for the treatment of advanced-stage keratoconus with steepcurvature. 2013; 24 (4):296-301.

9. Wagoner MD, Smith SD, Rademaker WJ, Mahmood MA. Penetrating keratoplasty vs. epikeratoplasty for the surgical treatment of keratoconus". Journal of Refractive Surgery, 2001; 17 (2): 138-146.
10. Jones MNA, Armitage WJ, Ayliffe W, Larkin DF, Kaye SB. Penetrating and deep anterior lamellar keratoplasty for keratoconus: a comparison of graft outcomes in the United Kingdom. Invest Ophthalmol Vis Sci.; 2009; 50(12):5625-5629.

11. Reinhart WJ, Musch DC, Jacobs DS, Lee WB, Kaufman SC, Shtein RM. Deep anterior lamellar keratoplasty as an alternative to penetrating keratoplasty: a report by the American Academy of Ophthalmology. Ophthalmology; 2011; 118(1):209-218.

12. Barbara A, Shehadeh-Masha'our R, Zvi F, Garzozi HJ. Management of pellucid marginal degeneration with intracorneal ring segments. J. Refract. Surg. (2005) 21, 296298.

13. Fogla R, Padmanabhan P. Results of deep lamellar kerato-plasty using the big bubble technique in patients with keratoconus. Am J Ophthalmol; 2006; 141(2):254 -9.

14. Yusuf Ozerturk, Esin Sogutlu Sari, Anil Kubaloglu, Arif Koytak, David Pinero, Sibel Akyol. Comparison of deep anterior lamellar keratoplasty and intracorneal ring segment implantation in advanced keratoconus.J Cataract Refract Surg 2012;38:324-332.

15. Khalid Mahmoud, Muhammad Tariq Khan, Wasim Iqbal Chaudary, Amjad Saleem Sahi. Visual outcome after deep lamellar keratoplasty in keratoconus. J Ophthalmol, 2010.Vol 26 NO.4.

16. Javadi, M. A.; Sepehr Feizi, Hossein Jamali, Firooz Mirbabaee. Deep anterior lamellar keratoplasty using the big bubble technique in keratoconus. J Ophthalmic, 2009:4(1): 8-13.

17. Fogla, R. Deep anterior lamellar keratoplasty in the management of keratoconus. Indian journal of ophthalmology, 2013, 61 NO. 8.

18. Feizi Sepehr, Mohammad Ali Javadi, Hossein Jamali, and Firooz Mirbabaee, Deep Anterior Lamellar Keratoplasty in Patients with Keratoconus: Big-Bubble Technique Cornea 2010; 29:177-182.

19. Fontana L, Parente G, Tassinari G. Clinical outcomes after deep anterior lamellar keratoplasty using the big-bubble technique in patients with keratoconus. Am J Ophthalmol 2007; 143:117-24.

20. Smadja David, Joseph Colin, Ronald R. Krueger, Glauco R. Mello. Outcomes of 
Deep Anterior Lamellar Keratoplasty for Keratoconus: Learning Curve and Advantages of the Big Bubble Technique. Cornea 2012:1-5.

21. Kubaloglu A, Sari ES, Ünal M, Koytak A, Kurnaz E, Cinar Y, Ozertürk Y. Longterm results of deep anterior lamellar keratoplasty for the treatment of keratoconus. Am J Ophthalmol; 2011; 151:760 -767.

22. Sarnicola V, Toro $\mathbf{P}$, Gentile $\mathbf{D}$, Hannush SB. Descemetic DALK and predescemetic DALK: Outcomes in 236 cases of keratoconus. Cornea. 2010; 29:53-9.

23. Han DC, Mehta JS, Por YM, Htoon HM, Tan DT. Comparison of outcomes of lamellar keratoplasty and penetrating keratoplasty in keratoconus. Am J Ophthalmol; 2009; 148:744-51.

24. Ünal M, Bilgin B, Yucel I, Akar Y, Apaydin C. Conversion to deep anterior lamellar keratoplasty (DALK): learning curve with big-bubble technique. Ophthalmic Surg Lasers Imaging; 2010; 41:642-50.

25. Coskunseven E, Onder M, Kymionis GD, Diakonis VF, Arslan E, Tsiklis N. Combined Intacs and posterior chamber toric implantable collamer lens implantation for keratoconic patients with extreme myopia. Am J Ophthalmol.; 2007; 144:387-9.

26. Shabayek MH and Alio JL. Intrastromal corneal ring segment implantation by femto second laser for keratoconus correction. Opthalmology 2007: 114 (9) :1643-1652.

27. Sansanayudh W, Bahar I, Kumar NL, Shehadeh-Mashour R, Retenour R, Signal $\mathbf{N}$, Rootman DS. Intrastromal corneal ring segment SK implantation for moderate to severe keratoconus. J Cataract Refrac Surg, 2010:36 (1):110-113.

28. Coskunseven E., Jankov M.R., 2nd, Grentzelos M.A., Plaka A.D., Limnopoulou A.N., Kymionis G.D. Topography-guided transepithelial PRK after intracorneal ring segments implantation and corneal collagen CXL in a three-step procedure for keratoconus. J Refract Surg. 2013; 29:54-58.

29. Foroutan AR, Dastjerdi MH. Shiftingbubble sign in big-bubble technique in deep anterior lamellar keratoplasty. Cornea 2007; 26:117; author reply 117-18. 
تقييم الترقيع الطبقى الجزئى الأمامى العميق للقرنية مقابل زراعة حلقات دعاميه داخل سدى القرنية لعلاج المرحلة المتوسطة

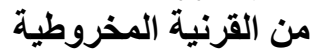

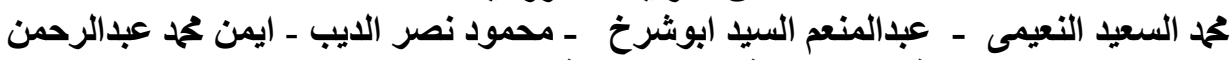

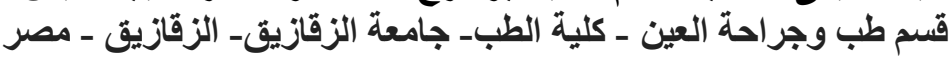

\section{الملخص العربيى}

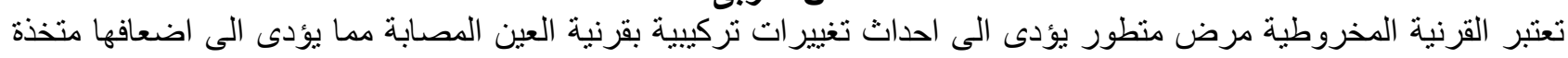

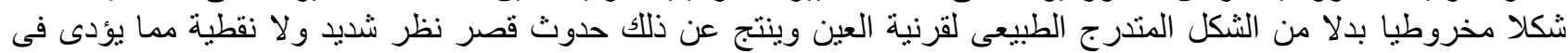

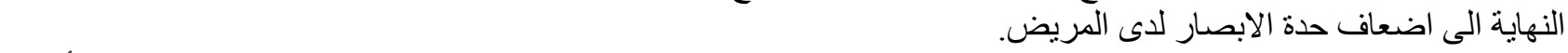
ونظر الان علاج القرنية المخروطية يعتمد على المرحلة التى تم تشخيص المرض فيها فقد تم تقسيم القرنيى المخروطية الى أربعة

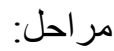

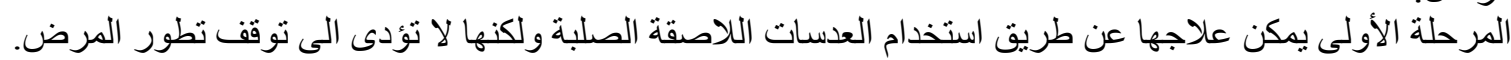

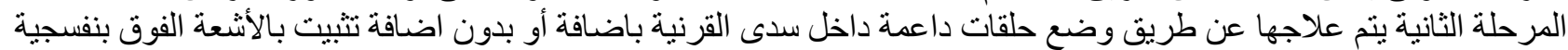

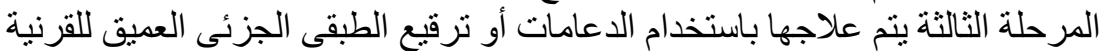

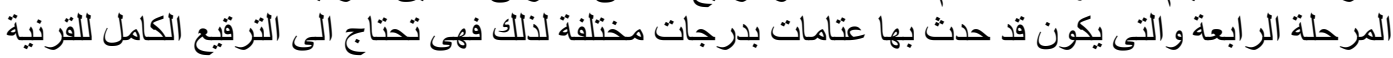

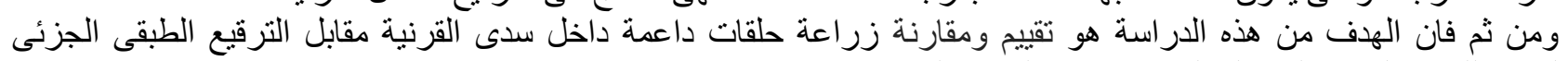

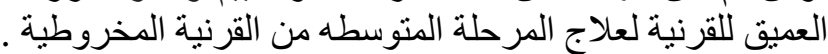
يعتبر الترقيع السطحى العميق للقرنية المخروطية أفضل من الترقيع الكامل للقرنية وذلك بسبب قلة نسبة حدوث ارتجاع لهذه القرنية. أهمية الدراسة والهدف منها: الهدف من هذه الرسالةهو ثقيبم ومقارنة الترقيع الطبقى الجزئى العميق للقرنبة مقابل زر اعة حلقات

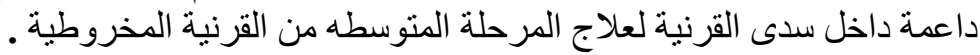
التصميم : در اسة مقارنة.

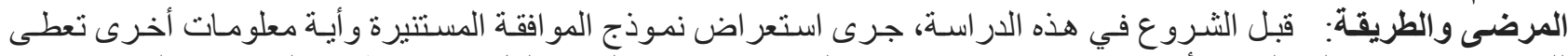

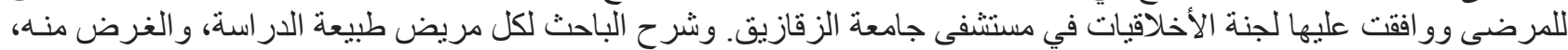

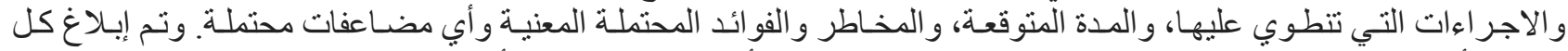

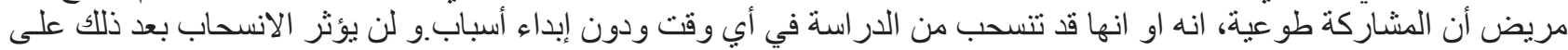

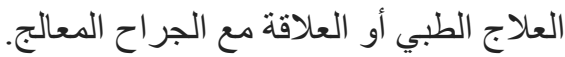

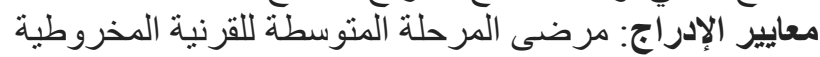

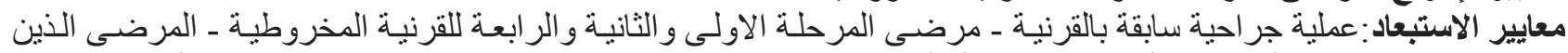

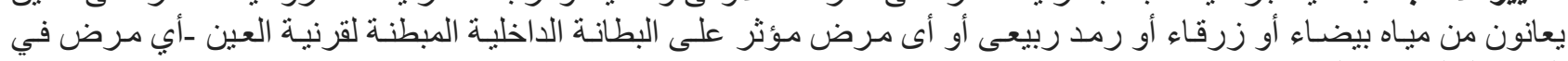

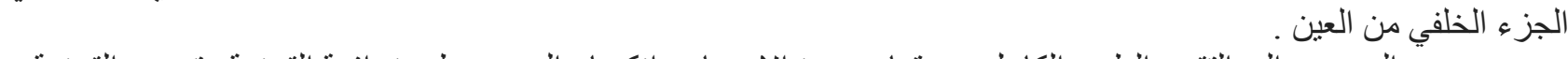
وخضع جميع المرضى إلى إلى التقييم الطبي الكامل من قياس حدة الابصـار و انكسـار العين و طبو غر افيـة القرنيـة وتحدب القرنيـة و ضغط العين و قاع العين وسمك القرنية.

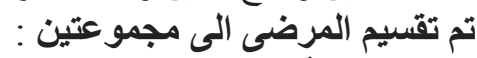

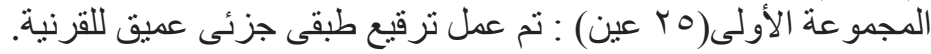

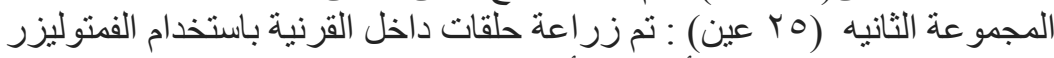

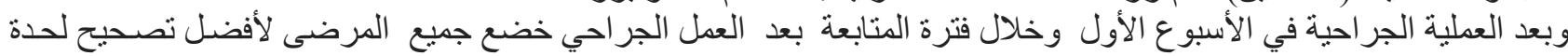

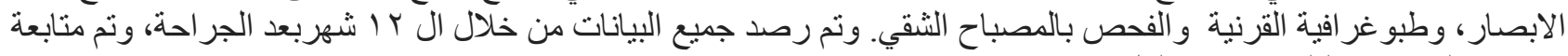

ومقارنة النتائج وتحليلها إحصائيا لكل مجمو عة الفية.

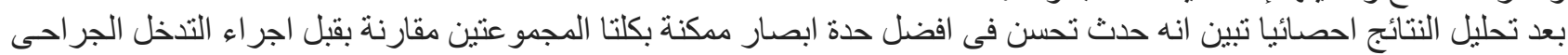

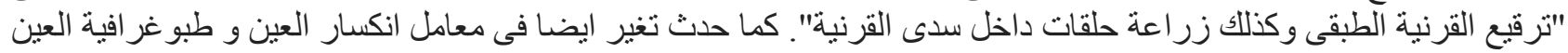

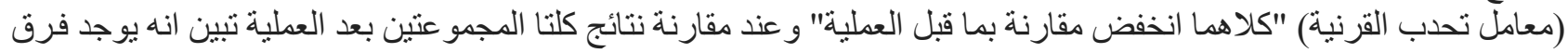

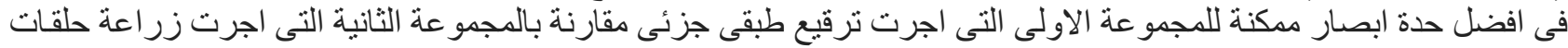

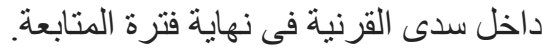

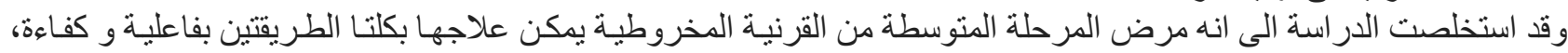

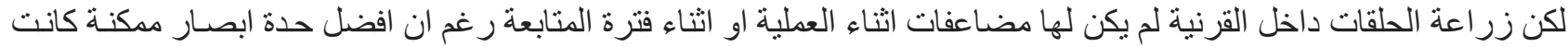

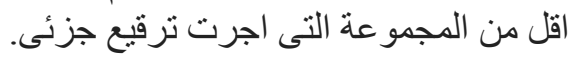

